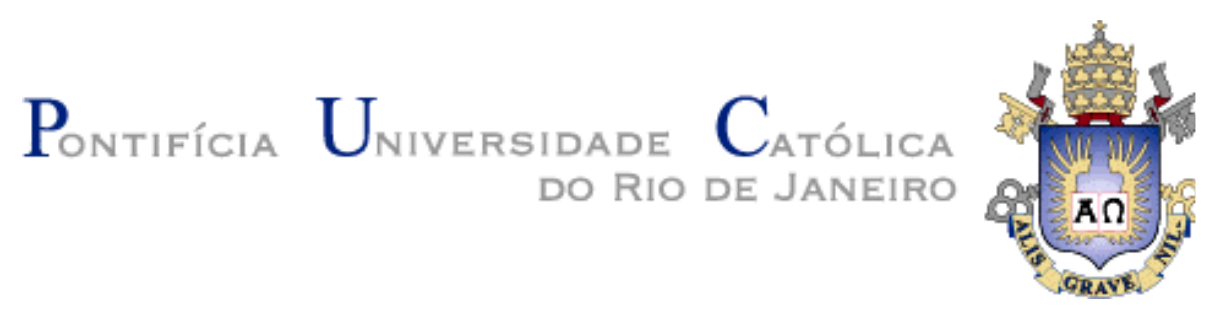

Uirá Kulesza

\title{
Uma Abordagem Orientada a Aspectos para o Desenvolvimento de Frameworks
}

Tese de Doutorado

Tese apresentada ao Programa de PósGraduação em Informática da PUC-Rio como requisito parcial para obtenção do grau de Doutor em Informática.

Orientador: Carlos José Pereira de Lucena 


\title{
Pontifícia Universidade Católlica $_{\text {a }}$ DO RIO DE JANEIRO

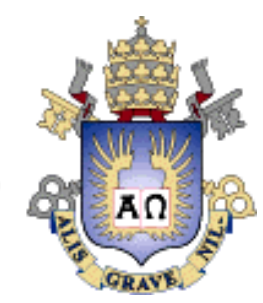

Uirá Kulesza

\section{Uma Abordagem Orientada a Aspectos para o Desenvolvimento de Frameworks}

\author{
Tese apresentada ao Programa de Pós- \\ graduação em Informática da PUC-Rio como \\ requisito parcial para obtenção do grau de \\ Doutor em Informática. Aprovada pela Comissão \\ Examinadora abaixo assinada.
}

Prof. Carlos José Pereira de Lucena Orientador Departamento de Informática - PUC-Rio

Prof. Arndt von Staa Departamento de Informática - PUC-Rio

Prof. Renato Cerqueira Departamento de Informática - PUC-Rio

Prof. Paulo Henrique Monteiro Borba Centro de Informática - UFPE

Prof. Paulo Cesar Masiero Instituto de Ciências Matemática e de Computação - USP

Prof. José Eugenio Leal Coordenador Setorial do Centro Técnico Científico - PUC-Rio

Rio de Janeiro 25 de Abril de 2007 
Todos os direitos reservados. É proibida a reprodução total ou parcial do trabalho sem autorização da universidade, do autor e dos orientadores.

\section{Uirá Kulesza}

Graduou-se no Curso de Bacharelado em Ciência da Computação da Universidade Federal de Campina Grande (UFCG) em 1998. Obteve o título de Mestre em Ciência da Computação na Universidade de São Paulo (USP) em 2000. Atuou como coordenador de projetos, engenheiro de processo e engenheiro de software no Centro de Estudos e Sistemas Avançados do Recife (CESAR) e na Qualiti Software Processes de 2000 a 2002. É pesquisador na área de Desenvolvimento de Software Orientado a Aspectos no Laboratório de Engenharia de Software (LES) da PUC-Rio, desde 2003.

Ficha Catalográfica

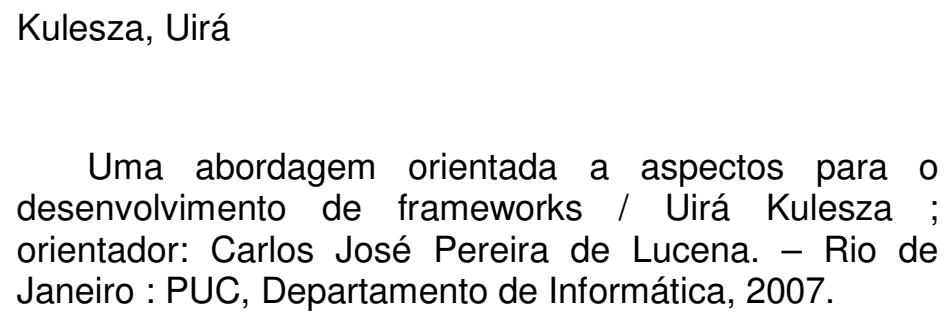

205 f. ; $30 \mathrm{~cm}$

Tese (Doutorado em Informática) - Pontifícia Universidade Católica do Rio de Janeiro, Rio de Janeiro, 2007.

Inclui referências bibliográficas.

1. Informática - Teses. 2. Frameworks orientados a objetos. 3. Desenvolvimento de software orientado a aspectos. 4. Arquiteturas de família de sistemas. 5. Desenvolvimento generativo. 6. Projeto de software. I. Lucena, Carlos José Pereira de. II. Pontifícia Universidade Católica do Rio de Janeiro. Departamento de Informática. III. Título.

CDD: 004 


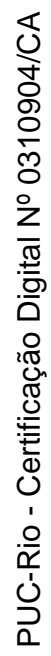

Ao nosso bondoso Pai que nunca deixa de nos guiar nas nossas caminhadas. 


\section{Agradecimentos}

Durante os anos de doutorado na PUC-Rio, tive a oportunidade de não apenas aprender a encarar problemas desafiadores e complexos vivenciados pela comunidade de engenharia de software, mas também desfrutar da convivência, conhecimento e amizade de vários parceiros de pesquisa do Laboratório de Engenharia de Software (LES) e de outras universidades.

Professor Lucena me acolheu no LES, me manteve sempre motivado e focado na minha pesquisa. Ele também transmitiu tremendo conhecimento na arte de coordenar e sincronizar atividades de vários grupos de pesquisa atuando em um mesmo laboratório. Passado todos esses anos no Rio, posso dizer que tive não apenas um excelente orientador para enveredar na carreira científica, mas ganhei sobretudo a amizade de uma grande pessoa.

Alessandro Garcia (o "Véio") foi um parceiro constante e ativo de vários trabalhos de pesquisa durante os últimos anos. Meu doutorado se iniciou como spin-off da sua tese, e as direções seguintes que trilhei receberam sempre inestimável contribuição de sua parte. A parceira continou mesmo com sua partida para Lancaster, e eu posso dizer com toda certeza que ele tem uma presença "crosscutting" nesse trabalho. Agradeço também pela oportunidade de realização conjunta dos estudos empíricos quantitativos com outros membros do grupo de Aspectos da PUC-Rio.

Vander Alves também contribuiu significativamente para os resultados alcançados nesse trabalho. Tivemos excelentes e estimulantes discussões durante o desenvolvimento de nossas respectivas teses. Juntamente com Alberto Costa Neto e professor Paulo Borba, todos do Software Productivity Group (SPG) da UFPE, eles contribuíram para validar e evoluir a abordagem proposta na tese, aplicando-a em um estudo de caso.

Roberta Coelho e Elder Cirilo do grupo de aspectos da PUC-Rio também tiveram participação substancial e ativa na realização desse trabalho. Roberta na 
definição e validação da abordagem com colaboração nos estudos de caso, e Elder na implementação da ferramenta que contempla o modelo generativo proposto na tese e que é o tema central da sua dissertação de mestrado. Raoni Kulesza e Klessis Dias estão usando muito dos conceitos propostos na tese no desenvolvimento de frameworks orientado a aspectos para diferentes domínios.

Cláudio Sant'Anna (o "Baiano") me acolheu desde o meu primeiro dia de trabalho no grupo de Aspectos do LES, foi um amigo extremamente presente e trouxe sempre sugestões diárias construtivas para evolução do trabalho. Ele foi também parceiro constante em várias outras pesquisas realizadas durante o doutorado.

Diversos outros membros/amigos do grupo de aspectos do LES contribuíram indiretamente na realização do trabalho e também para o meu crescimento profissional: (i) professor Arndt von Staa ofereceu várias sugestões e novas perpectivas do trabalho, sobretudo em relação ao uso da tecnologia de geradores de aplicação; (ii) Christina Chavez da UFBA, doutora e ex-pesquisadora do grupo de aspectos da PUC-Rio, trouxe sempre sugestões/questionamentos e ofereceu novas direções para a realização do trabalho; e finalmente, (iii) Cidiane Lobato e Eduardo Magno compartilharam comigo os resultados de suas pesquisas e estimularam discussões "cruzadas" de nossos trabalhos.

Outros pesquisadores que agradeço pelos trabalhos conjuntos ou sugestões oferecidas em algum momento da realização desse trabalho são: André van der Hoek, Awais Rashid, Birgit Geppert, David Weiss, Don Cowan, Jean-Pierre Briot, Itana Gimenes, Krzysztof Czarnecki, Kyo Kang, Lyrene Fernandes, Marcílio Mendonça, Michal Antkiewicz, Mira Mezini, Paulo Alencar, Peter Kim, Thais Batista, Toacy Cavalcante.

Foi um prazer aprender e colaborar com todos vocês, amigos e parceiros, e espero que a colaboração se perpetue ainda por vários anos. E a amizade dure para sempre!

Agradeço a todos os membros da banca pelas sugestões oferecidas para 
melhoria do texto apresentado nesse documento e novas perspectivas de reflexão do trabalho.

Agradeço as agências de fomento FAPERJ e CNPq que forneceram o apoio financeiro necessário para realização desse trabalho.

Não poderia também deixar de agradecer a imensa "família" de amigos que me apoiaram imensamente durante esses anos de doutorado.

Roberta Coelho, minha esposa querida, sempre me motivou e me manteve animado nos momentos difíceis de realização da tese. Ao seu lado encarar o doutorado foi muito mais fácil e tranquilo.

Vera Menezes me ofereceu seu sorriso diário e o apoio necessário a qualquer problema adicional encontrado no doutorado. Obrigado Verita, por tudo! Sentirei imensa saudade sua no ambiente de trabalho.

Sílvia Passos, minha madrinha e amiga, me ofereceu sempre seu lar e sua divertida e agradável companhia durante vários finais de semana. Ricardo Rocha e Renata foram também amigos importantes durante todos esses anos de caminhada no Rio. Juba e Dilene foram companhias constantes em programas de lazer, shows e choppinhos de relaxamento nos finais de semana.

Vários outros amigos do LES e da PUC tornaram a minha vida de doutorando em diferentes fases mais divertida e fácil, entre eles (juntamente com os já citados acima): Akeo, Anarosa, Andrew, Bruno, Caculé, Carol, Chicão, Daflon, Dani "Gaúcha”, Dani Kussel, Daniel, Felipe, Firmo, Guilherme, Guga, Hana, Ivan, Karla, Léo Cunha, LF, Maíra, Mariela, Michel, Miriam, Pádua, Renato Maia, Ricardo Choren, Ricardo Gralhoz, Rodrigo “Alagoano”, Vagner, Viviane.

Agradeço também a toda equipe e amigos do Centro de Pastoral Anchieta da PUC-Rio por todos os ensinamentos durante esses anos que contribuíram imensamente para a minha formação humana/social. As sementes plantadas por vocês, estão certamente germinando. 
Outros amigos fundamentais nessa caminhada foram a família Coelho/Cordeiro, família Mitsunaga/Kulesza e todos do DreamTeam (turma de graduação da UFCG).

Finalmente agradeço meu pai, minha mãe, minha esposa e cada um dos meus irmãos (incluindo Nandinho), pelo apoio incondicional, sorrisos, pensamento positivo, aprendizado mútuo e atenção dedicada durante todos esses anos. Sem vocês ao lado tudo isso ficaria sem sentido! Obrigado por me fazerem enxergar e sentir os verdadeiros valores da vida! 


\section{Resumo}

Kulesza, Uirá. Uma Abordagem Orientada a Aspectos para o Desenvolvimento de Frameworks. Rio de Janeiro, 2007. 205p. Tese de Doutorado - Departamento de Informática, Pontifícia Universidade Católica do Rio de Janeiro.

Esse trabalho propõe uma abordagem sistemática para o desenvolvimento de frameworks usando técnicas orientadas a aspectos (OA). O objetivo central da abordagem é melhorar a capacidade de extensão e configuração de frameworks orientados a objetos $(\mathrm{OO})$ para diferentes cenários de reutilização, através de uma melhor gerência de suas características. A abordagem é composta por: (i) um conjunto de diretrizes para o projeto e implementação de frameworks usando programação orientada a aspectos; e (ii) um modelo generativo usado para a instanciação automática do framework e suas variabilidades $\mathrm{OO}$ e OA. As diretrizes propõem a definição de um conjunto de pontos de junção de extensão (EJPs - extension join points) no código do framework, os quais podem ser usados para estender a funcionalidade básica do framework através da implementação de aspectos de extensão. Tais aspectos são responsáveis pela implementação de características transversais opcionais, alternativas ou de integração demandadas por usuários do framework. A abordagem é demonstrada com a implementação / refatoração de 3 frameworks OO pertencentes a diferentes domínios de aplicação. Uma avaliação da abordagem por meio de um estudo qualitativo e um estudo quantitativo é também apresentada. Finalmente, diversas lições aprendidas e discussões resultantes da experiência de uso da abordagem são descritas.

\section{Palavras-chave}

Frameworks Orientados a Objetos; Desenvolvimento de Software Orientado a Aspectos; Arquiteturas de Família de Sistemas; Desenvolvimento Generativo; Projeto de Software. 


\section{Abstract}

Kulesza, Uirá. An Aspect-Oriented Approach to Framework Development. Rio de Janeiro, 2007. 205p. PhD Thesis - Computer Science Department, Pontifical Catholical University of Rio de Janeiro.

This work proposes a systematic approach to framework development which relies on the use of aspect-oriented (AO) techniques. The main goal of the approach is to improve the extensibility and configurability of objectoriented (OO) frameworks. It is composed of: (i) a set of guidelines to design and implement frameworks using aspect-oriented programming; and (ii) a generative model which allows the automatic instantiation of the framework and its respective $\mathrm{OO}$ and $\mathrm{AO}$ variabilities. Our guidelines propose the definition of extension join points (EJPs) in the framework code, which can be used to extend the framework basic functionality by means of extension aspects. The extension aspects are responsible for implementing optional, alternative and integration crosscutting features required by the framework users. Since such aspects can be automatically unplugged from the framework code, our approach makes it easier to customize the framework to specific needs. Three cases studies are presented to illustrate the applicability of our approach to the development of frameworks from different domains. The approach is also evaluated through both a qualitative and a quantitative study. Finally, several lessons learned and discussions resulting from the use of the approach are described.

\section{Keywords}

Object-Oriented Frameworks; Aspect-Oriented Software Development; System Family Architectures; Generative Development; Software Design. 


\section{Sumário}

1 Introdução 20

$\begin{array}{ll}\text { 1.1. Problema } & 21\end{array}$

1.2. Limitações das Abordagens Atuais 22

1.3. Solução Proposta 23

1.4. Organização do Texto 24

2 Abordagens de Desenvolvimento de Família de Sistemas 26

2.1. Frameworks Orientados a Objetos 26

2.1.1. Problemas de Modularização 27

2.1.1.1. Framework JUnit: Um Exemplo 28

2.1.1.2. Complexidade de Colaboração entre Objetos 29

2.1.1.3. Dificuldade de Modularização de Características Opcionais 31

2.1.1.4. Composições Transversais na Integração de Frameworks 35

2.2. Desenvolvimento de Software Orientado a Aspectos 38

2.2.1. AspectJ 39

2.2.2. Interfaces Transversais 40

2.3. Desenvolvimento Generativo 42

2.4. Potencial de Integração entre as Abordagens 44

2.5. Sumário 47

3 Uma Abordagem Orientada a Aspectos para o Desenvolvimento de $\begin{array}{ll}\text { Frameworks } & 48\end{array}$

3.1. Diretrizes para Implementação de Frameworks com Aspectos 48

3.1.1. Pontos de Junção de Extensão (EJPs) 48

3.1.2. Núcleo do Framework e Aspectos de Extensão 49

3.2. Um Modelo Generativo Orientado a Aspectos 51

3.3. Fluxo de Atividades da Abordagem 52

3.4. Sumário 56 
4 Diretrizes para o Projeto e Implementação de Frameworks usando Orientação a Aspectos $\quad 57$

4.1. Implementando EJPs em AspectJ 57

4.1.1. Documentação Visual de EJPs e Aspectos de Extensão 57

4.1.2. Estrutura de EJPs 59

4.1.3. Especialização de EJPs 61

4.1.4. Contratos de EJPs 62

4.2. Implementando Aspectos de Extensão em AspectJ 66

4.2.1. Variabilidades em Aspectos de Extensão 68

4.3. Sumário 70

5 Um Modelo Generativo Orientado a Aspectos 71

5.1. Visão Geral 71

5.2. Engenharia de Domínio: Definição do Modelo Generativo OA 73

5.2.1. Especificação do Modelo de Arquitetura 73

5.2.2. Especificação do Modelo de Características 75

5.2.3. Especificação do Modelo de Configuração 77

5.2.4. Codificação de Templates 84

5.3. Engenharia de Aplicação: Instanciação da Arquitetura $O A$

5.3.1. Escolha de Variabilidades no Modelo de Característica 86

5.3.2. Escolha de Relações Transversais no Modelo de Característica 87

5.3.3. Algoritmo de Instanciação de Arquiteturas OA 88

5.4. Sumário 91

6 Estudos de Casos 92

6.1. Framework Measurement 92

6.1.1. Núcleo do Framework 92

6.1.2. Pontos de Junção de Extensão 95

6.1.3. Aspectos de Integração 96

6.1.3.1. Composição com o Framework GUI 97

6.1.3.2. Composição com o Framework de Estatística 103

6.1.3.3. Composição com o Framework de Persistência 107

6.1.4. Modelo Generativo OA 110 
6.2. AspectT 113

6.2.1. Núcleo do Framework 113

6.2.2. Pontos de Junção de Extensão 116

6.2.3. Aspectos do Núcleo 117

6.2.3.1. Adaptação 117

6.2.3.2. Autonomia 120

6.2.4. Aspectos de Extensão 123

6.2.4.1. Aprendizagem 123

6.2.4.2. Mobilidade 128

6.2.4.3. Colaboração 133

6.2.5. Modelo Generativo 134

6.3. Linha de Produto de Jogos J2ME 137

6.3.1. Núcleo do Framework 137

6.3.2. Pontos de Junção de Extensão 138

6.3.3. Aspectos de Extensão 141

6.3.4. Modelo Generativo 143

$\begin{array}{ll}\text { 6.4. Sumário } & 146\end{array}$

7 Análise dos Estudos de Caso, Discussões e Lições Aprendidas 147

7.1. Benefícios da Abordagem 147

7.2. Estudo de Composição de Frameworks 150

7.2.1. Estudo Qualitativo 150

7.2.1.1. Análise Qualitativa das Soluções OO 151

7.2.1.2. Análise Qualitativa das Soluções OA 156

7.2.2. Estudo Quantitativo 161

7.2.2.1. As Métricas Utilizadas 162

7.2.2.2. Análise e Discussão dos Resultados 164

7.3. Discussões e Lições Aprendidas 168

7.3.1. Composição e Interação de Aspectos de Extensão 168

7.3.2. Documentação de EJPs 170

7.3.3. Casos de Uso de Extensão 171

7.3.4. Modelagem e Estabilidade de EJPs 172

7.3.5. Estratégias de Adoção de Linhas de Produto de Software 173 
8 Trabalhos Relacionados $\quad 176$

8.1. Interfaces Transversais (XPIs) 176

8.2. Abordagens para Implementação de Famílias de Software $\quad 177$

8.2.1. Programação Orientada a Características 177

8.2.2. Método de Decomposição Horizontal 179

8.2.3. Frameworks Transversais 180

8.2.4. Aspectos de Especialização 182

8.2.5. Framed Aspects 182

8.2.6. Abordagem Extrativa de Linha de Produtos 183

8.3. Abordagens para Instanciação de Frameworks e Linhas de Produto184

8.3.1. Abordagem para Instanciação de Frameworks $\bigcirc \bigcirc$

8.3.2. Pure::Variants 186

9 Conclusões e Trabalhos Futuros $\quad 187$

9.1. Contribuições 188

9.2. Trabalhos em Andamento e Futuros 189

$\begin{array}{ll}\text { Referências } & 191\end{array}$

Apêndice I Tradução de Termos $\quad 201$

Apêndice II Estudo Quantitativo 202 


\section{Lista de Figuras}

Figura 1. Diagrama de Classes do framework JUnit ................................229

Figura 2. Implementação do monitoramento de testes no JUnit.................31

Figura 3. Implementação de propriedades de extensão no JUnit............... 33

Figura 4. Composição de propriedades de extensão no JUnit................... 34

Figura 5. Exemplo de Composição entre Frameworks ............................... 37

Figura 6. Aspecto FaultHandler ............................................................... 40

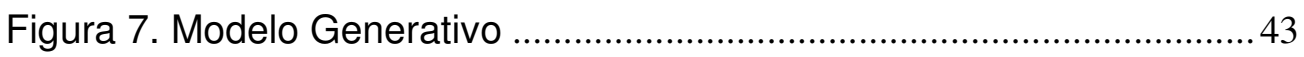

Figura 8. Elementos de Implementação da Abordagem Proposta ............50

Figura 9. Atividades de Engenharia de Domínio da Abordagem ...............53

Figura 10. Atividades de Engenharia da Aplicação da Abordagem...........53

Figura 11. Diagrama de Classes e Aspectos do JUnit ...............................58

Figura 12. Aspecto EJP TestExecutionEvents ....................................60

Figura 13. Aspecto de Extensão RepeatAllTests ......................................61

Figura 14. Exemplo de Especialização de EJP ...........................................62

Figura 15. Exemplo de Contratos de EJPs no Contexto do JUnit .............65

Figura 16. Aspecto de Variabilidade ActiveTest ...................................67

Figura 17. Aspecto de Variabilidade ActiveAlltest.Suite..................67

Figura 18. Aspecto de Variabilidade RepeatedTests ............................6 69

Figura 19. Exemplos de Especialização de Aspectos de Extensão .......... 70

Figura 20. Visão Geral dos Elementos do Modelo Generativo .................. 72

Figura 21. Modelo Generativo do Framework JUnit................................... 83

Figura 22. Template Test Sui teTemplate..............................................85

Figura 23. Instância do Modelo de Característica ...................................... 87

Figura 24. Definição de Relações Transversais ....................................... 88

Figura 25. Classes e Aspectos Gerados para uma Configuração do JUnit

Figura 26. Diagrama de Classes do Framework Measurement.................93

Figura 27. Diagrama de Seqüência do Framework Measurement ............ 94

Figura 28. Exemplo de Instância do Framework Measurement................. 95

Figura 29. Aspecto EJP MeasurementEvent s.......................................96 


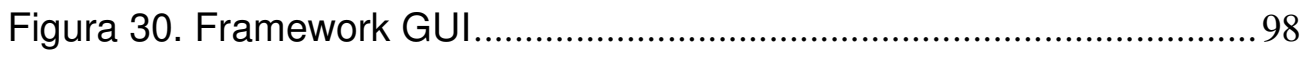

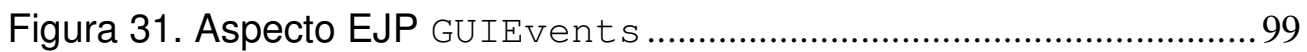

Figura 32. Integração entre os frameworks Measurement e GUI ............. 100

Figura 33. Aspecto de Integração Measurement GUIAspect .................. 101

Figura 34. Aspecto BeerCanMeasurementGUIAspect........................... 102

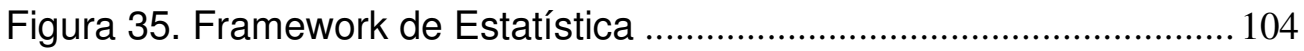

Figura 36. Integração entre os FWs Measurement, GUI e Estatística .... 105

Figura 37. Aspecto Mea surement GUIStat ist icAspect ..................... 106

Figura 38. Integração entre os FWs GUI, de Estatística e de Persistência 108

Figura 39. Aspecto de Integração Pers istenceAspect ........................ 109

Figura 40. Aspecto de Integração BeerCanPersistenceAspect ....... 109

Figura 41. Modelo Generativo da Composição dos Frameworks ............. 112

Figura 42. Núcleo do Framework AspectT .................................................. 114

Figura 43. Diagrama de Seqüência do Framework AspectT ...................... 115

Figura 44. Exemplo de Instância do Framework AspectT ........................... 116

Figura 45. Aspecto EJP Aspect TEvents ....................................................116

Figura 46. Aspecto do Núcleo Adaptation .....................................................118

Figura 47. Aspecto do Núcleo Adaptation .............................................119

Figura 48. Aspecto do Núcleo Autonomy ................................................... 120

Figura 49. Aspecto do Núcleo Autonomy ………………........................ 122

Figura 50. Estrutura do Padrão de Projeto Learning ................................... 124

Figura 51. Aspecto de Extensão Learning .............................................. 125

Figura 52. Aspecto de Extensão ChairLearning ................................... 127

Figura 53. Aspecto de Extensão Mobility …………………………........ 129

Figura 54. Aspecto de Extensão Mobility ……………………………...... 131

Figura 55. Aspecto de Extensão ChairMobility ……………………....132

Figura 56. Aspecto de Extensão de Colaboração Chair ........................... 134

Figura 57. Modelo Generativo do AspectT ............................................... 136

Figura 58. Diagrama de Classes do núcleo do Rain of Fire ...................... 138

Figura 59. Diagrama de Seqüência do Rain of Fire .................................... 139

Figura 60. Arquitetura da Linha de Produto Rain of Fire ........................... 140 
Figura 61. Aspecto de Variabilidade Clouds ............................................. 142 Figura 62. Modelo de Configuração da Linha de Produto do Rain of Fire 145

Figura 63. Métricas de Separação de Interesses 165

Figura 64. Métricas de Tamanho, Acoplamento e Coesão.......................... 166 


\section{Lista de Tabelas}

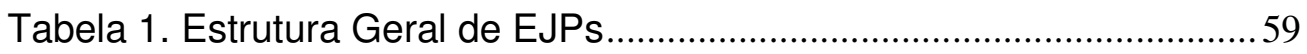

Tabela 2. Contratos Internos do Framework..............................................63

Tabela 3. Contratos de Extensão do Framework. .......................................64

Tabela 4. Elementos do Modelo de Configuração....................................... 78

Tabela 5. Regras de Mapeamento entre Características e Elementos de

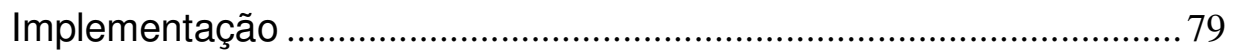

Tabela 6. Elementos do Modelo de Configuração do JUnit. .......................82

Tabela 7. Análise dos Benefícios das Diretrizes da Abordagem ............. 149 Tabela 8. Soluções OO para Composição de Fluxo de Controle de FWs

Tabela 9. Soluções OO para Lacuna entre Frameworks. 153

Tabela 10. Soluções $\mathrm{OO}$ para Composição de Funcionalidades de Entidades 154

Tabela 11. Avaliação de Propriedades das Soluções 00 155

Tabela 12. Soluções OA para Composição de Fluxo de Controle de FWs

Tabela 13. Soluções OA para Lacuna entre Frameworks 158

Tabela 14. Soluções $O A$ para Composição de Funcionalidades de Entidades 159

Tabela 15. Análise de Propriedades das Soluções OA 160

Tabela 16. Métricas de Acoplamento. 163

Tabela 17. Métricas de Tamanho 163

Tabela 18. Métricas de Separação de Interesses (Sol). 163

Tabela 19. Métrica de Coesão 164 Tabela 20. Valores Absolutos para Métricas de Separação de Interesses

Tabela 21. Valores para Métricas de Acoplamento, Coesão e Tamanho164

Tabela 22. Valores Coletados para Métricas - Versão OO. 203

Tabela 23. Valores Coletados para Métricas - Versão OA 205 


\section{Lista de Siglas e Abreviaturas}

\begin{tabular}{|c|c|}
\hline ADL & Architecture Description Language \\
\hline AWT & Abstract Window Toolkit \\
\hline DAO & Data Access Object \\
\hline DG & Desenvolvimento Generativo \\
\hline DSL & Domain Specific Language \\
\hline DSOA & Desenvolvimento de Software Orientado a Aspectos \\
\hline EJP & Extension Join Point ou Ponto de Junção de Extensão \\
\hline EMF & Eclipse Modeling Framework \\
\hline FOP & Feature Oriented Programming \\
\hline GUI & Graphical User Interface \\
\hline JET & Java Emitter Template \\
\hline $\mathrm{J} 2 \mathrm{ME}$ & Java 2 Micro Edition \\
\hline LPS & Linha de Produto de Software \\
\hline POA & Programação Orientada a Aspecto \\
\hline OA & Orientado a Aspecto \\
\hline $\mathrm{OO}$ & Orientado a Objeto \\
\hline SoI & Separação de Interesses \\
\hline UML & Unified Modeling Language \\
\hline XPI & Crosscutting Interface ou Interface Transversal \\
\hline
\end{tabular}

